Check for updates

Cite this: RSC Adv., 2018, 8, 37949

Received 3rd August 2018

Accepted 20th October 2018

DOI: $10.1039 / c 8 r a 06556 j$

rsc.li/rsc-advances

\section{Superior ion release properties and antibacterial efficacy of nanostructured zeolites ion-exchanged with zinc, copper, and iron $\dagger$}

\author{
Shaojiang Chen, (D) a John Popovich, ${ }^{b}$ Wenwen Zhang, ${ }^{a}$ Collin Ganser, (D) ${ }^{c}$ \\ Shelley E. Haydel (DD ${ }^{* b c}$ and Dong-Kyun Seo (D) *a
}

\begin{abstract}
Antimicrobial zeolites ion-exchanged with inexpensive transition metal ions (such as zinc, copper, and iron) are critically important for a broader adoption of the materials for public health applications. Due to the high surface area and small particle sizes, nanozeolites are particularly promising in enhancing the efficacy of the zeolite-based antimicrobial materials. By using highly-crystalline nanostructured zeolites (FAU) with textural mesoporosity, we report a comprehensive study on the materials characteristics of zinc-, copper-, and iron-ion exchanged nanozeolites, the ion release properties, and antibacterial efficacy against methicillin-resistant Staphylococcus aureus (MRSA), as well as a comparison of the properties to those obtained for the corresponding microsized zeolites. Superior ion release properties were observed for both zinc and copper ion-exchanged nanostructured zeolite $\mathrm{X}$, with ion release up to $73 \%$ for zinc and $36 \%$ for copper of their initial loadings, as compared to $50 \%$ and $12 \%$, respectively, for the corresponding microsized zeolites, validating the importance of nanostructuring for enhanced ion diffusion through zeolite pore channels. The 2 hours minimum bactericidal concentration (MBC) in saline for the copper ion-exchanged nanostructured zeolite $X$ was $32 \mu \mathrm{g} \mathrm{mL}{ }^{-1}$, half the corresponding microsized zeolite $X$ MBC of $64 \mu \mathrm{g} \mathrm{mL}^{-1}$. Our results established nanostructured zeolite $X$ as a superior host material for metal ion-based antimicrobials, with the aforementioned improvements for copper-exchanged nanozeolites compared to previous studies.
\end{abstract}

\section{Introduction}

Nanosized or nanostructured zeolites, collectively termed "nanozeolites", are of great interest in various industrial applications, such as catalysis and gas separation, with the premise that their high external surface area and short diffusion length would enhance their functionalities in those applications where molecular diffusion is a critical factor. The performance of nanozeolites has also been studied for new emerging applications such as sensors, drug delivery and antimicrobials. ${ }^{1-4}$ Transition metal ions, such as $\mathrm{Ag}^{+}, \mathrm{Zn}^{2+}, \mathrm{Cu}^{2+}, \mathrm{Fe}^{2+}$, and others, have been recognized as effective antimicrobials with minimal toxicity to humans. ${ }^{5-10}$ Zeolites are an ideal material to host and release the metal ions because of their controllable ion exchange properties and the high thermal and chemical

${ }^{a}$ School of Molecular Sciences, Arizona State University, Tempe, AZ 85287, USA. E-mail:DSeo@asu.edu; Tel: +1-480-727-7789

${ }^{b}$ Biodesign Institute Center for Immunotherapy, Vaccines and Virotherapy, Arizona State University, Tempe, AZ 85287, USA. E-mail: Shelley.Haydel@asu.edu; Tel: +1480-727-7234

${ }^{c}$ School of Life Sciences, Arizona State University, Tempe, AZ 85287, USA

$\uparrow$ Electronic supplementary information (ESI) available. See DOI: 10.1039/c8ra06556j stabilities desired for industrial production processes. ${ }^{\mathbf{1 1}}$ Indeed, zeolites loaded with antimicrobial metal ions consistently demonstrate activity against a broad spectrum of microorganisms. ${ }^{12-16}$ Ion release from zeolites occurs when the ions in zeolite diffuse through the zeolite body to the external surface, so the increased surface area and nanoscopic dimension of nanozeolites are desirable for an enhanced ion release performance. The antimicrobial applications of the nanozeolites are particularly timely in the age of continuously emerging antimicrobial resistance, where the fight to prevent the spread of infection is critical and cannot be understated.

While silver has been well studied for its antimicrobial efficacy, other more affordable metals are generating interest for antimicrobial applications. Zinc oxide nanoparticles ${ }^{17}$ and zinc ion-exchanged zeolites ${ }^{\mathbf{1 2}}$ have been exploited for their antimicrobial activity. In addition to its antimicrobial properties, zinc aids wound healing when applied topically, ${ }^{\mathbf{1 8 , 1 9}}$ making it clinically efficacious in wound care and management. While use of copper as an antimicrobial has existed for millennia, there are widespread efforts to enhance its antimicrobial activity and expand its use in healthcare settings and environmental applications. $^{\mathbf{2 0 , 2 1}}$ Although iron is not frequently investigated as an antimicrobial, there is some evidence that ferrous iron $\left(\mathrm{Fe}^{2+}\right)$ released by ion-exchanged clays contributes to antibacterial 
activity. ${ }^{7}$ Iron and copper ions can cause oxidative damage to lipids, proteins, and DNA via Fenton and Haber-Weiss reactions, potentially leading to indirect, metal-induced antibacterial activity. ${ }^{22}$ At the molecular level, copper and zinc ions can damage cytoplasmic dehydratase enzymes, leading to rapid enzymatic inactivation and bacterial cell death. ${ }^{23,24}$

Despite the important benefits of the antimicrobial zeolites ion-exchanged with $\mathrm{Cu}^{2+}, \mathrm{Zn}^{2+}$, or $\mathrm{Fe}^{2+}$, comprehensive studies which examine the correlation between the innate material properties of zeolite particles, especially of nanozeolites, and their antimicrobial efficacy are lacking. Furthermore, the studies rarely investigate material concentration-dependence on antimicrobial efficacy. In perhaps the most comprehensive study, Demirci et al. ${ }^{12}$ showed that their $\mathrm{Cu}^{-}$and $\mathrm{Zn}$-ion-exchanged microsized zeolite $\mathrm{X}$ samples have inhibitory activity against Staphylococcus aureus, Bacillus cereus, Escherichia coli, and Pseu-

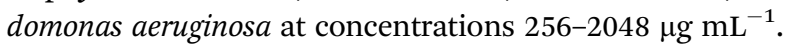

In our previous work, ${ }^{16}$ we demonstrated that $\mathrm{Ag}^{+}$-ionexchanged highly-crystalline nanostructured zeolite $\mathrm{X}$ offers superior release kinetics and rapid antibacterial activity compared to their microsized counterparts. ${ }^{\mathbf{1 6}}$ For example, Demirci et al. ${ }^{12}$ revealed $\mathrm{Ag}^{+}$-ion-exchanged micron-sized zeolite $\mathrm{X}$ at concentrations of $32-64 \mu \mathrm{g} \mathrm{mL} \mathrm{m}^{-1}$ displayed $24 \mathrm{~h}$ inhibitory activity against $S$. aureus in trypticase soy broth (TSB), ${ }^{12}$ while in our work, the $\mathrm{Ag}^{+}$-ion-exchanged nanostructured zeolites inhibited methicillin-resistant Staphylococcus aureus (MRSA) at $16 \mu \mathrm{g} \mathrm{mL}{ }^{-1}$ in the same medium. Furthermore, the $\mathrm{Ag}^{+}$-ionexchanged nanostructured zeolites showed a minimum bactericidal concentration (MBC; $>99.9 \%$ population reduction) of 1 $\mu \mathrm{g} \mathrm{mL}{ }^{-1}$ after $2 \mathrm{~h}$ in water. ${ }^{16}$ Particle size of zeolites significantly affected ion release, as shown in our studies: ${ }^{16}$ zeolite $\mathrm{X}$ aggregates with a primary particle size of $24 \mathrm{~nm}$ show $43 \% \mathrm{Ag}$ ion release within a few minutes while only $18 \%$ could be released from $2 \mu \mathrm{m}$-sized zeolites during the same time. The work demonstrated that nanostructured zeolites, with their high surface area and short ion diffusion path lengths, rapidly release silver ions and kill bacteria, compared to the microsized counterparts. In expanding the development of high performance antimicrobials with anticipated cost benefits, we report preparation of zinc, copper, and iron ion-exchanged nanostructured zeolites and their materials properties, superior ion release behavior, and antibacterial activities against MRSA.

\section{Results and discussion}

\section{Zeolite structures, morphologies and pore characteristics}

The $\mathrm{Si} / \mathrm{Al}$ ratios were estimated to be 1.06 for mZeo and 1.47 for nZeo from X-ray diffraction studies (see below) and thus their theoretical ion exchange capacities (IECs) are 5.28 and 4.58 meq. $\mathrm{g}^{-1}$, respectively. The extent of ion exchange was determined by measuring the amount of the metal ions left after ion exchange in the solution. The results are shown in Table 1 where the parent zeolites are labeled with the prefix "Na-", while the ion-exchanged zeolites are with the prefix that indicates the metal used for ion exchange. For the microsized zeolite (mZeo), the ion exchange was 53.6, 69.1 and 50.5\% of its IEC for $\mathrm{Zn}^{2+}$, $\mathrm{Cu}^{2+}$ and $\mathrm{Fe}^{2+}$ ions, respectively. For the nanosized zeolite (nZeo), the corresponding values were $50(\mathrm{Zn}), 58.0(\mathrm{Cu})$ and $41.2 \%(\mathrm{Fe})$. With the assumption that the chemical compositions remain the same except for the exchanged ions, the relative amounts of $\mathrm{Zn}, \mathrm{Cu}$ and $\mathrm{Fe}$ to the original zeolites are calculated to be 9.02, 11.2 and $7.37 \mathrm{wt} \%$, respectively, for mZeo, which are equivalent to $1.38,1.77$ and $1.32 \mathrm{mmol} \mathrm{g}^{-1}$, respectively. The corresponding values for nZeo are $7.32(\mathrm{Zn}), 8.26(\mathrm{Cu})$ and $5.21 \mathrm{wt} \%$ (Fe), which correspond to $1.12,1.30$ and $0.93 \mathrm{mmol} \mathrm{g}^{-1}$, respectively. A recent study by Redfern et al. on nanosized zeolite $\mathrm{X}(\mathrm{Si} / \mathrm{Al}=1.2)$ indicated that $75 \%$ of sodium ions was exchanged with copper ions, resulting in $11 \mathrm{wt} \% \mathrm{Cu}$ content. ${ }^{25}$ For the microsized zeolite $\mathrm{X}(\mathrm{Si} / \mathrm{Al}=1.25)$, up to $18 \mathrm{wt} \% \mathrm{Zn}$ and $15 \mathrm{wt} \% \mathrm{Cu}$ have been reported after ion exchange at room temperature by Demirci et al., although the detailed analysis was not given. ${ }^{12}$ In any event, the extent of ion exchange in our experiments was somewhat lower than the those values.

Powder X-ray diffraction (PXRD) patterns of parent zeolites and the ion-exchanged zeolites are shown in Fig. 1 where the patterns are grouped for the sake of easy comparison of the peak intensities and positions. All the samples exhibit Bragg reflection peaks whose positions match those calculated for an FAU zeolite crystal structure. ${ }^{\mathbf{2 6}}$ The Bragg peaks are sharp for both parent Na-mZeo and Na-nZeo, indicating a high crystallinity of the samples. The size of Na-nZeo crystallites is estimated to be $24 \mathrm{~nm}$ based on the peak broadening using Scherrer equation, as reported in our previous work. ${ }^{16}$ Additionally, the $\mathrm{Si} / \mathrm{Al}$ ratios of the Na-nZeo and Na-mZeo are 1.47

Table 1 Exchanged metal ion contents and pore characteristics of the zeolites

\begin{tabular}{|c|c|c|c|c|c|c|c|}
\hline \multirow[b]{2}{*}{ Sample } & \multirow{2}{*}{$\begin{array}{l}\text { Ion exchange } \\
\text { (equiv.\%) }\end{array}$} & \multicolumn{2}{|c|}{$\begin{array}{l}\text { Transition metal } \\
\text { content }^{a}\end{array}$} & \multirow[b]{2}{*}{$S_{\mathrm{BET}}\left(\mathrm{m}^{2} \mathrm{~g}^{-1}\right)$} & \multirow[b]{2}{*}{$S_{\text {micro }}\left(\mathrm{m}^{2} \mathrm{~g}^{-1}\right)$} & \multirow[b]{2}{*}{$V_{\text {total }}\left(\mathrm{cm}^{3} \mathrm{~g}^{-1}\right)$} & \multirow[b]{2}{*}{$V_{\text {micro }}\left(\mathrm{cm}^{3} \mathrm{~g}^{-1}\right)$} \\
\hline & & $\left(\mathrm{mmol} \mathrm{g}^{-1}\right)$ & (wt\%) & & & & \\
\hline Na-mZeo & - & 0 & 0 & 695 & 663 & 0.34 & 0.31 \\
\hline Na-nZeo & - & 0 & 0 & 783 & 674 & 0.54 & 0.31 \\
\hline Zn-mZeo & $53.6(0.5)$ & 1.38 & 9.02 & 605 & 501 & 0.34 & 0.23 \\
\hline Cu-nZeo & $58.0(0.8)$ & 1.30 & 8.26 & 561 & 425 & 0.46 & 0.20 \\
\hline Fe-mZeo & $50.5(0.2)$ & 1.32 & 7.37 & 438 & 262 & 0.29 & 0.12 \\
\hline Fe-nZeo & $41.2(0.2)$ & 0.93 & 5.21 & 471 & 272 & 0.49 & 0.13 \\
\hline
\end{tabular}

${ }^{a}$ Standard deviations are shown in parentheses. 

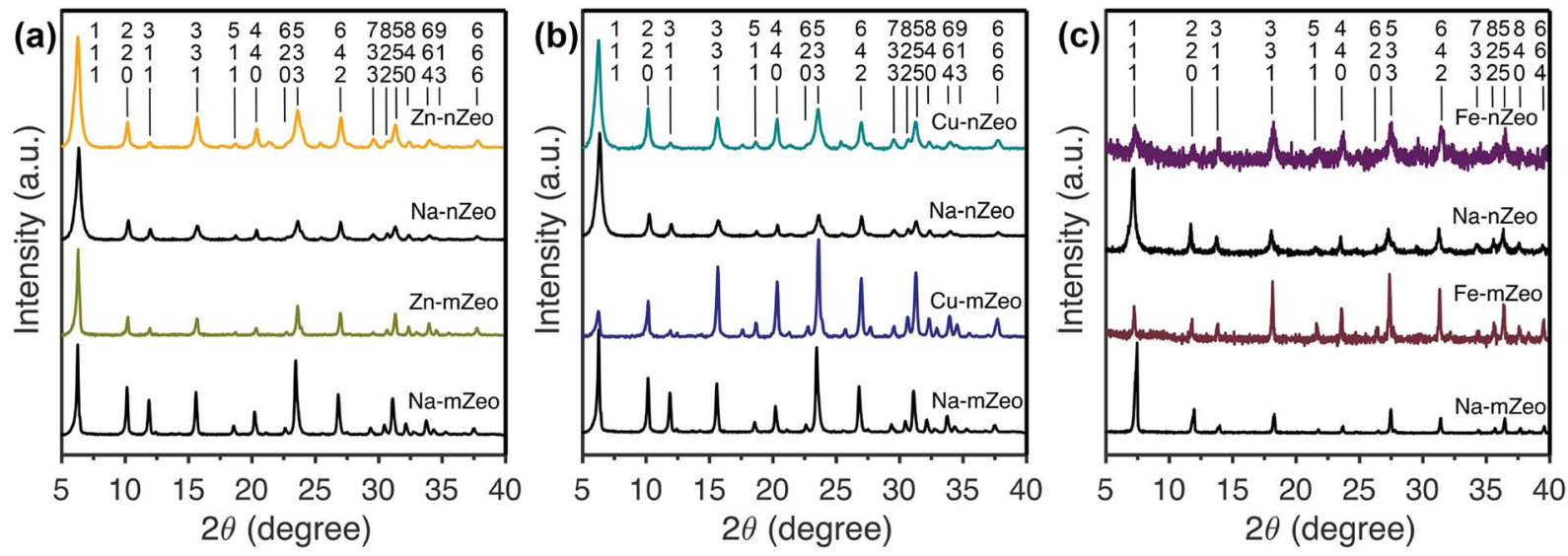

Fig. 1 PXRD patterns of parent Na-mZeo and Na-nZeo samples along with the (a) Zn-mZeo and Zn-nZeo (Cu Ka), (b) Cu-mZeo and Cu-nZeo $(\mathrm{Cu} \mathrm{K \alpha})$, and (c) Fe-mZeo and Fe-nZeo (Co K $\alpha$ ) samples. The numbers in patterns are Miller indices for the Bragg peaks.

and 1.06, respectively, based on the unit cell constants refined with a cubic FAU structure $(a=24.866$ and $24.999 \AA$, respectively).

Since there were no appreciable changes in the Bragg peak positions after the ion exchange, the unit cell dimensions are conserved for all the samples during the ion exchange process. Likewise, negligible changes in peak intensities would be expected when the exchanged $\mathrm{Na}$ ions and the replacing transition metal ions have similar preferences in their locations in zeolite structure. This is indeed the case for Zn-mZeo, Zn-nZeo (Fig. 1a) and Cu-nZeo (Fig. 1b). However, the PXRD pattern of $\mathrm{Cu}-\mathrm{mZeo}$ exhibits different relative peak intensities compared to the parent Na-mZeo (Fig. 1b), indicating that the $\mathrm{Cu}$ ions prefer different locations in mZeo. ${ }^{27}$ For example, the three strongest Bragg peaks in the PXRD of Na-mZeo, (111), (220) and (331), are located at $2 \theta=6.3,23.6$, and $10.2^{\circ}$, respectively, while for Cu-mZeo, the peaks (533) at $23.6^{\circ},(331)$ at $15.6^{\circ}$, and (555) at $31.2^{\circ}$ are the strongest. In contrast, there is no obvious change in relative peak intensities between the PXRD patterns of $\mathrm{Cu}-$
nZeo and Na-nZeo (Fig. 1b), which is consistent with a previous report. ${ }^{25}$ For both Fe-nZeo and Fe-mZeo (Fig. 1c), the ion exchange led to a distinct change in Bragg peak intensities. Moreover, the Bragg peaks of Fe-nZeo are broader than the parent Na-nZeo, indicating partial collapse of zeolite structure during the iron ion exchange. Given the importance of the ion distribution in understanding the ion release performance, efforts to determine metal ion positions by Rietveld refinement are underway and will be published separately.

The morphology of zeolite samples before and after ion exchange was investigated by SEM (Fig. 2). Na-mZeo exhibits a typical isotropic crystal shape of the FAU zeolite with average particle sizes of 1-3 $\mu \mathrm{m}$ and sharp crystal facets (Fig. 2a), while Na-nZeo shows submicron-sized particles with highly textured surfaces (Fig. 2e). TEM images of the Na-nZeo indicate that the submicron-sized particles observed in the SEM studies are made up of primary nanoparticles with plate-like morphologies (Fig. S1†). Most of the nanoparticles are in the range of 10$30 \mathrm{~nm}$ in length, in agreement with the particle size $(24 \mathrm{~nm})$
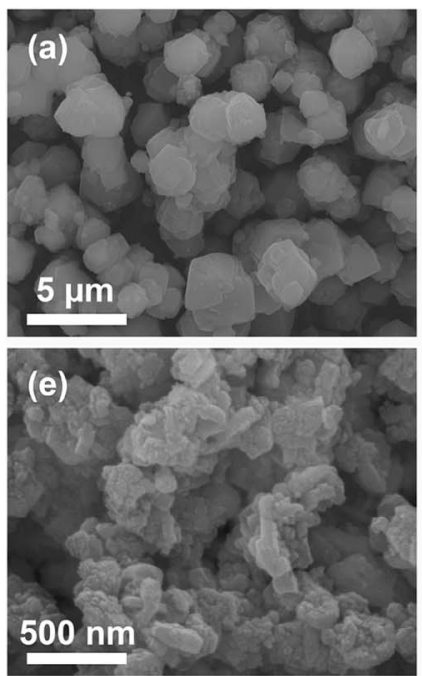
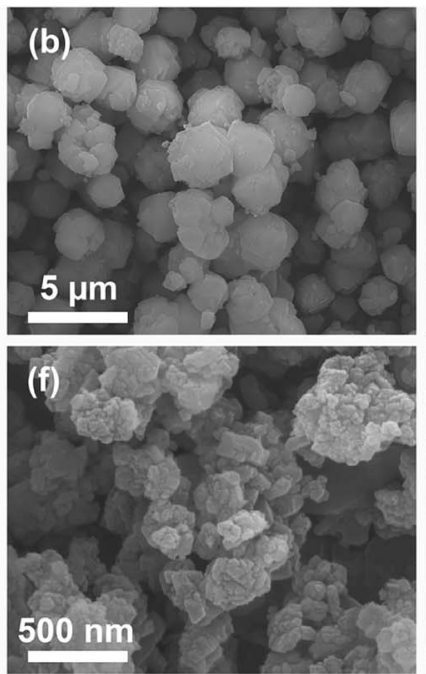
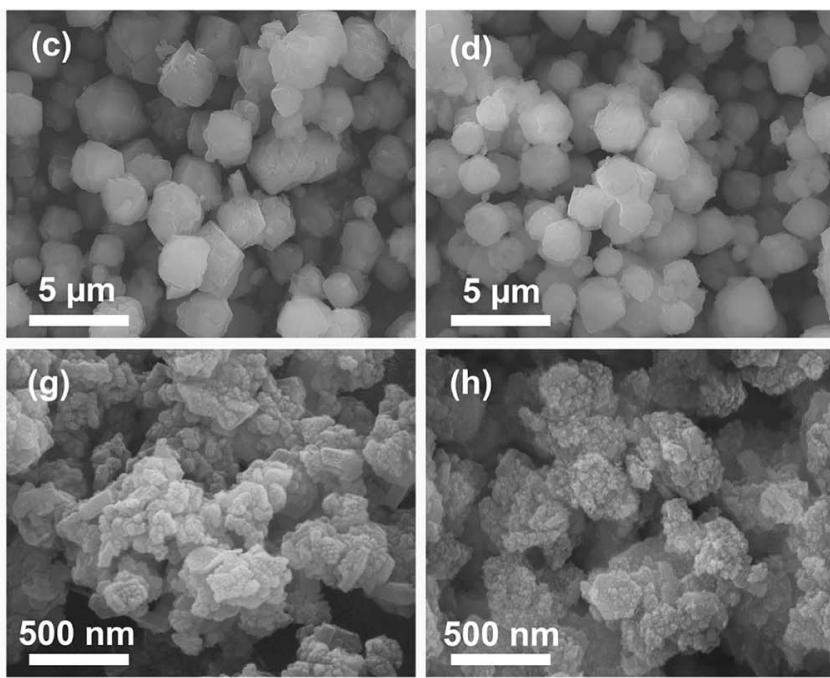

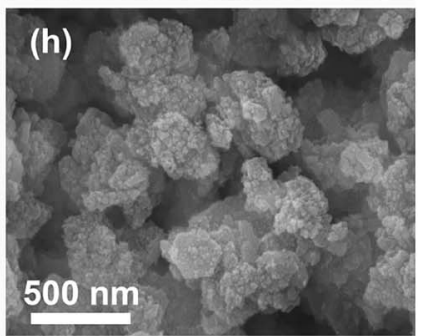

Fig. 2 Scanning electron microscopic (SEM) images of parent (a) Na-mZeo and (e) Na-nZeo samples; (b) Cu-mZeo and (f) Cu-nZeo samples; (c) Zn-mZeo and (g) Zn-nZeo samples; (d) Fe-mZeo and (h) Fe-nZeo samples. 

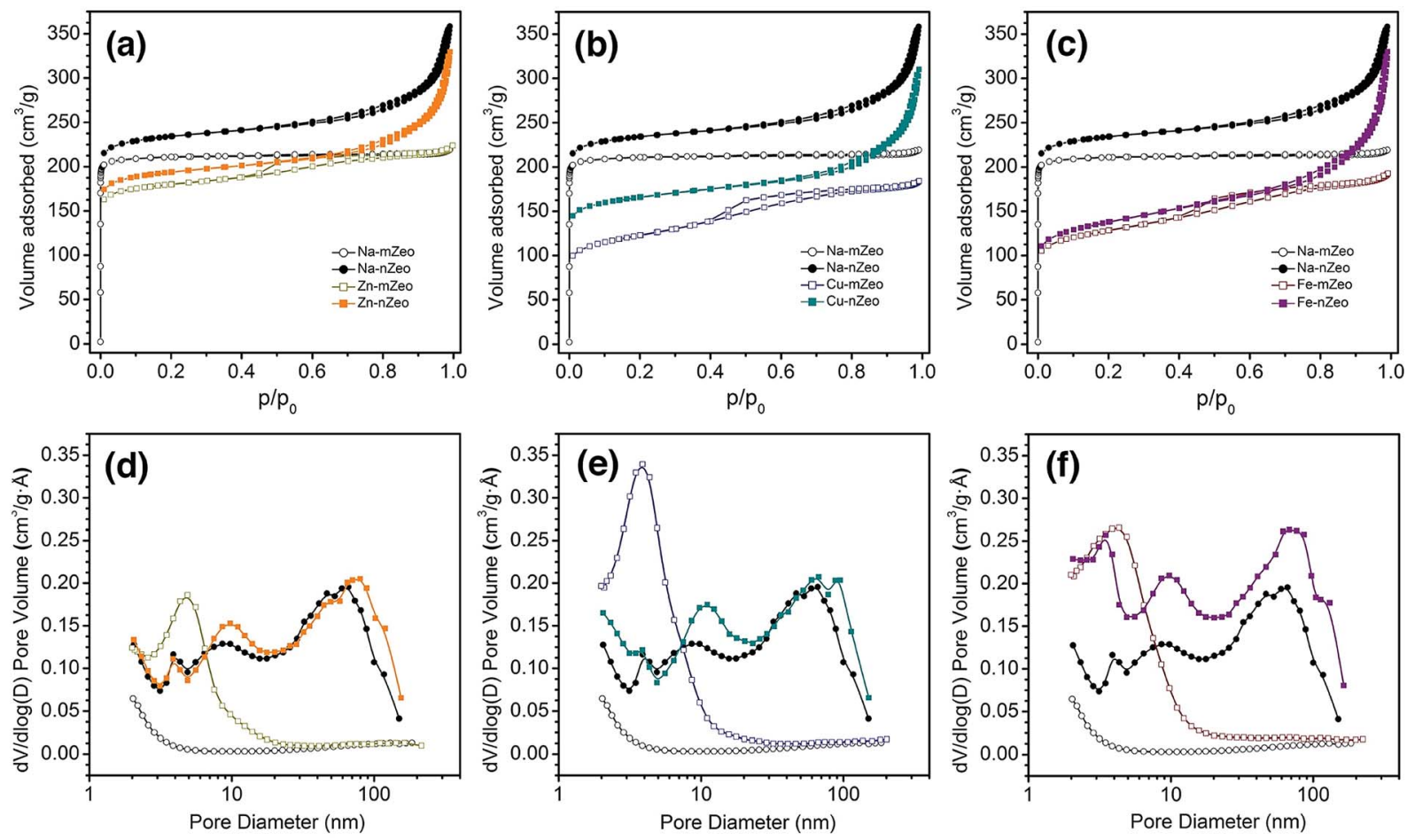

Fig. 3 Nitrogen sorption isotherms $(a-c)$ and pore width distributions $(d-f)$ of parent Na-mZeo and Na-nZeo along with the Zn-mZeo and ZnnZeo ( $a$ and d); Cu-mZeo and Cu-nZeo (b and e); Fe-mZeo and Fe-nZeo (c and f).

estimated from the PXRD studies, although some have a lateral dimension as large as $100 \mathrm{~nm}$. Morphologies of the ionexchanged mZeo and nZeo samples are similar to their parent forms (Fig. 2), indicating that the ion exchange process preserved the original morphology. These results are consistent with our previous studies on silver ion-exchanged zeolites. ${ }^{16}$

Since pore characteristics of zeolites affect metal ion release and thereby the antibacterial activities, nitrogen sorption measurements were performed to characterize pore size and distribution of the zeolite samples. As shown in Fig. 3a-c, the Na-mZeo exhibits a classical type I isotherm of microporous materials, characterized by immediate uptake at low relative pressure region $\left(p / p_{0}<0.01\right)$ followed by horizontal adsorption and desorption branches. In contrast, the Na-nZeo shows a type IV isotherm, with a large initial gas intake and a large H1-type hysteresis (Fig. 3a-c). As shown in Table 1, the micropore volume of Na-nZeo $\left(0.31 \mathrm{~cm}^{3} \mathrm{~g}^{-1}\right)$ is equivalent to that of NamZeo, revealing a comparable crystallinity. The hysteresis indicates a presence of mesoporosity which must be textural and from the aggregate morphology of the nZeo observed in SEM (Fig. 2) and TEM (Fig. S1 $\dagger$ ) images. The Na-nZeo has a total pore volume of $0.54 \mathrm{~cm}^{3} \mathrm{~g}^{-1}$, much larger than $0.34 \mathrm{~cm}^{3} \mathrm{~g}^{-1}$ for Na-mZeo, due to the additional textural porosity. The BJH pore width distribution calculated from desorption branch shows the mesopores as well as small macropores in the range of 10100 nm (Fig. 3d-f).

After the ion exchange, Zn-mZeo, Cu-mZeo, and Fe-mZeo exhibit a new hysteresis loop in $p / p_{0}=0.40-0.80$ in their isotherms (Fig. $3 \mathrm{~d}-\mathrm{f}$ ). The hysteresis loops are responsible for the peaks centered around $4 \mathrm{~nm}$ in $\mathrm{BJH}$ pore width distribution calculated from adsorption branch (desorption branch has artificial peaks, Fig. S1a-c †). Zn-nZeo, Cu-nZeo, and Fe-nZeo show the same type of isotherms as the parent Na-nZeo, but with less gas adsorption at low relative pressure region $\left(p / p_{0}<\right.$ 0.01) (Fig. 3d-f). The BJH total pore volume of Zn-mZeo and ZnnZeo are 0.34 and $0.49 \mathrm{~cm}^{3} \mathrm{~g}^{-1}$, respectively, which are comparable to 0.34 and $0.54 \mathrm{~cm}^{3} \mathrm{~g}^{-1}$ of the parent Na-mZeo and Na-nZeo, respectively (Table 1). However, the micropore volumes of Zn-mZeo and Zn-nZeo are 0.23 and $0.25 \mathrm{~cm}^{3} \mathrm{~g}^{-1}$, respectively, which are lower than the values before the ion exchange. The decrease in the microporosity may indicate a partial amorphization of zeolite framework during the ion exchange process. It has been previously reported that $\mathrm{Zn}$ ionexchanged zeolite $\mathrm{X}$ undergoes dealumination of framework and subsequent re-organization to remove the resulting vacancies generated when subjected to dehydration process (for example, the degassing at $300{ }^{\circ} \mathrm{C}$ under vacuum for the gas sorption analysis). ${ }^{28}$ Metal ion size is unlikely a contributing factor to the lowered micropore volumes as $\mathrm{Zn}$ ions have smaller ionic radii than Na ions (0.74 and $1.02 \AA$, respectively, with $\mathrm{CN}=6) \cdot{ }^{29}$

The micropore volume was reduced more severely after $\mathrm{Cu}$ ion exchange than after $\mathrm{Zn}$ ion exchange $\left(0.11 \mathrm{~cm}^{3} \mathrm{~g}^{-1}\right.$ for $\mathrm{Cu}-$ mZeo and $0.20 \mathrm{~cm}^{3} \mathrm{~g}^{-1}$ for $\mathrm{Cu}-\mathrm{nZeo}$ ), while the total pore volumes were reduced only slightly (Table 1). Partial amorphization could have occurred during the ion exchange process, as in the case of $\mathrm{Zn}$ ion-exchanged zeolites. However, PXRD patterns showed that the crystallinity was retained after $\mathrm{Cu}$ ion exchange, and hence the loss of micropore volume may not be entirely due to collapse of zeolite structure, but rather due to 
degassing-mediated reduction of $\mathrm{Cu}^{2+}$ to $\mathrm{Cu}^{+}$and subsequent clustering of the metal ions that block the micropores. ${ }^{30}$ Unlike $\mathrm{Cu}$ ion exchange, $\mathrm{Fe}$ ion exchange reduced the micropore volumes in both Fe-mZeo $\left(0.12 \mathrm{~cm}^{3} \mathrm{~g}^{-1}\right)$ and Fe-nZeo $\left(0.13 \mathrm{~cm}^{3}\right.$ $\mathrm{g}^{-1}$ ) (Table 1). Similar to $\mathrm{Cu}$ ion-exchanged zeolites, amorphization might have occurred during the ion exchange process, as micropore volumes of $0.12 \mathrm{~cm}^{3} \mathrm{~g}^{-1}$ for Fe-mZeo and 0.13 for FenZeo were observed (Table 1). The decreased micropore volume could also be attributed to vacuum heating, causing migration of iron species and subsequent clustering that blocks the micropores. $^{31}$

\section{Ion release characteristics of ion-exchanged zeolites}

Release characteristics of zinc, copper, and iron ions from the ion-exchanged zeolites were studied in terms of release efficiency in amount and time. Fig. 4 shows the time-dependent metal ion release from ion-exchanged zeolite samples in saline solution $(0.9 \% \mathrm{NaCl} \mathrm{w} / \mathrm{v})$ at different contact times. The overall ion release amounts are larger for the zeolites exchanged
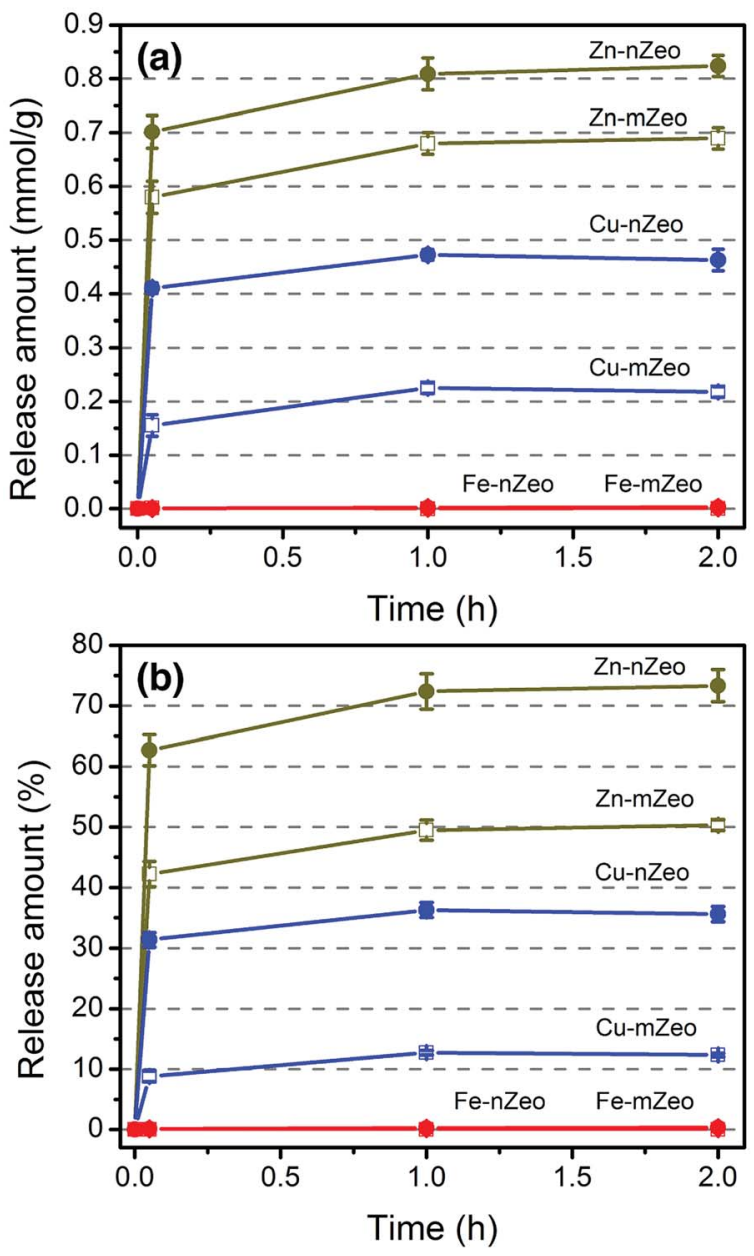

Fig. 4 Zinc, copper, and iron ion release curves from ion-exchanged zeolites in saline solution $(0.9 \% \mathrm{NaCl} ; \mathrm{w} / \mathrm{v})$ at different contact times; (a) absolute release amount in $\mathrm{mmol} \mathrm{g}^{-1}$; (b) release amount in percentage of total metal ion loading; the concentration of the ionexchanged zeolites was $1.0 \mathrm{mg} \mathrm{mL}^{-1}$. with zinc ions than with copper ions. It has been indicated that $\mathrm{Zn}^{2+}$ is bound to the FAU framework less strongly than $\mathrm{Cu}^{2+}$ through local charge neutralization, ${ }^{32}$ consistent with the previous observations that the self-diffusion is faster for $\mathrm{Zn}^{2+}$ than $\mathrm{Cu}^{2+}$ in microchannels of zeolite $\mathrm{X}^{33,34}$ As shown in Fig. 4a and Table S1, $\uparrow$ most of the exchangeable ions were released within $3 \mathrm{~min}$ for the $\mathrm{Cu}$ and $\mathrm{Zn}$ ion-exchanged zeolites, while no Fe ion release was observed for either Fe-mZeo or Fe-nZeo after $2 \mathrm{~h}$. Zn-nZeo consistently released more zinc ions than Zn-mZeo despite lower zinc loading of $1.12 \mathrm{mmol} \mathrm{g}^{-1}$, in comparison to $1.38 \mathrm{mmol} \mathrm{g}^{-1}$ for Zn-mZeo (Table S1; $\dagger$ Fig. 4b). The release amount at $2 \mathrm{~h}$ was $0.81 \mathrm{mmol} \mathrm{g}^{-1}$ for Zn-nZeo, which is $73.3 \%$ of the total loading (Table S1; $\uparrow$ Fig. $4 \mathrm{~b}$ ). In contrast, the corresponding loading and release values for $\mathrm{Zn}-\mathrm{mZeo}$ were $0.68 \mathrm{mmol} \mathrm{g}^{-1}$ and 50.3\%, respectively (Table $\mathrm{S} 1 ; \dagger$ Fig. $4 \mathrm{~b}$ ). Meanwhile, $\mathrm{Cu}-\mathrm{nZeo}$ released more copper ions than $\mathrm{Cu}-\mathrm{mZeo}$ during the $2 \mathrm{~h}$ observation period. At equilibrium, copper ion release was $0.47 \mathrm{mmol} \mathrm{g}^{-1}$ for $\mathrm{Cu}-\mathrm{nZeo}$, or $36.3 \%$ of the total loading (Table $\mathrm{S} 1 ; \dagger$ Fig. $4 \mathrm{~b}$ ). In contrast, copper ion release was $0.23 \mathrm{mmol} \mathrm{g}^{-1}$ for $\mathrm{Cu}-\mathrm{mZeo}$, which is half of the respective value for $\mathrm{Cu}-\mathrm{nZeo}$, and $\mathbf{1 2 . 3 \%}$ of the total loaded copper (Table S1; $\dagger$ Fig. 4b). These results are consistent with our previous Ag ionexchanged zeolite studies, whereby the nanostructured zeolite released more silver ions than microsized zeolite despite the same silver loading. ${ }^{16}$ In any event, superior release kinetics was apparent from our studies for nanostructured zeolite $\mathrm{X}$ with Ag, $\mathrm{Zn}$, and $\mathrm{Cu}$ ions. It is worth mentioning that Demirci et al. reported the concentration of copper ion released from their ionexchanged microsized zeolite $\mathrm{X}$ samples in TSB would correspond to over $100 \mathrm{wt} \%$ release. ${ }^{12}$ However, a previous report on the $\mathrm{Na} / \mathrm{Cu}$ ion-exchange isotherm for zeolite $\mathrm{X}$ implies that zeolite $\mathrm{X}$ retains $60 \%$ of $\mathrm{Cu}^{2+}$ ions in a $\mathrm{Na}^{+}$solution, ${ }^{32}$ and thus a complete release of the ions would have been challenging.

Release of iron ions was not observed for either Fe-mZeo and Fe-nZeo, indicating strong interactions between iron ions and the zeolite framework. The iron ions might be chemically anchored to the zeolite framework via strong Si-O-Fe and Al-OFe bridges. ${ }^{31,35}$ Alternatively, the iron species may exist as hydroxide or oxide forms, making them unexchangeable. ${ }^{31}$ Another possibility is that during the ion release experiments, the ferrous ions were oxidized to ferric ions which are not soluble in water. However, it is unlikely that iron would exist as part of zeolite framework by replacing the Al sites without any further treatment. ${ }^{36}$

For $\mathrm{Zn}$ and $\mathrm{Cu}$, therefore, the superior ion release characteristics of the ion-exchanged nZeo over their mZeo counterparts must be associated with the morphology (i.e., smaller primary particle sizes and larger surface areas) of the former. Not only can smaller particle sizes reduce the diffusion time due to the shorter diffusion path length, but also larger external surface areas can provide more openings for the metal ions to come into or exit from the zeolite bodies. This enhancement effect would be more apparent for the ions that are bound strongly with the framework and with lower mobility. ${ }^{34}$ Indeed, $\mathrm{Cu}$-nZeo is observed to show a much superior ion release performance than $\mathrm{Cu}-\mathrm{mZeo}$, while the effect is less between $\mathrm{Zn}$ nZeo and Zn-mZeo. These results are consistent with the 
behavior of the zeolites exchanged with $\mathrm{Ag}$ ions in our previous study. ${ }^{16}$

\section{Antibacterial activities of the ion-exchanged zeolites}

Zn-nZeo and Zn-mZeo were incubated with mid-logarithmic phase MRSA to determine antimicrobial efficacy. The MBC of Zn-nZeo and Zn-mZeo against MRSA was $512 \mu \mathrm{g} \mathrm{mL}{ }^{-1}$ after $2 \mathrm{~h}$ incubation in saline (Table 2; Fig. 5). Although the experimental conditions were different, Demirci et al., ${ }^{\mathbf{1 2}}$ reported zinc ionexchanged microsized zeolites, at concentrations ranging from 512 to $2048 \mu \mathrm{g} \mathrm{mL} \mathrm{m}^{-1}$, exhibited $24 \mathrm{~h}$ inhibition in TSB. Neither Zn-nZeo or Zn-mZeo (at concentrations $\leq 2048 \mu \mathrm{g}$ $\mathrm{mL}^{-1}$ ) inhibited MRSA growth after $24 \mathrm{~h}$ incubation in cationadjusted Mueller Hinton broth (CAMHB) (Table 2). CAMHB is a nutrient-rich medium that contains excess organic molecules and anions with potential to act as neutralizers, preventing $\mathrm{Zn}$ ions from killing cells. In contrast to CAMHB, saline contains only sodium and chloride ions and thus $\mathrm{Zn}$ ions remain dissolved in the solution to kill cells. Bactericidal activity was the same for both Zn-nZeo and Zn-mZeo (Table 2; Fig. 5), despite ion release data showing a slightly larger amount of $\mathrm{Zn}$ ions released from Zn-nZeo compared to Zn-mZeo (Fig. 4). The enhanced $\mathrm{Zn}$ ion release capacity of the Zn-nZeo compared to Zn-mZeo (Fig. 4), coupled with similar antimicrobial efficacy while using less zinc ions (Table 2), is beneficial for reducing production costs. Although the killing activity of Zn-nZeo was less efficacious when compared to Ag-nZeo ${ }^{16}$ or Cu-nZeo (discussed below), $\mathrm{Zn}$ ions influence wound healing, ${ }^{18,19}$ and thus clinical applications could be advantageous.
After incubation of mid-logarithmic phase MRSA in saline for $2 \mathrm{~h}$, the $\mathrm{MBC}$ for $\mathrm{Cu}-\mathrm{nZeo}$ was $32 \mu \mathrm{g} \mathrm{mL} \mathrm{m}^{-1}$, which is half the MBC for Cu-mZeo (Table 2; Fig. 6). It is noted that despite a lower loading of $\mathrm{Cu}$ ions in $\mathrm{Cu}-\mathrm{nZeo}$ than in $\mathrm{Cu}-\mathrm{mZeo}$, the former released about twice as many $\mathrm{Cu}$ ions as the latter (Fig. 4; Table $\mathbf{S 1 \dagger}$ ). Such a material efficiency is valuable for reducing production costs, which is important for future applications. ${ }^{\mathbf{1 2}}$ The $\mathrm{Cu}$ ion-exchanged zeolites showed MBC values lower in saline than what was reported by Demirci et al. ${ }^{12}$ (24 h inhibition concentrations ranging from 1024 to $2048 \mu \mathrm{g} \mathrm{mL}^{-1}$ in TSB), although they lacked antibacterial activity when incubated with MRSA in CAMHB for $24 \mathrm{~h}$ (Table 2). Although standardized procedures are lacking for testing inorganic materials and comparisons must be made with caution, Cu-nZeo was similar to or better than existing Cu-loaded zeolites generated for antimicrobial applications. ${ }^{12,37,38}$ While the Cu-nZeo was less efficacious than Ag-nZeo, ${ }^{\mathbf{1 6}}$ copper is commonly used in contamination prevention and is cheaper than silver. The potential applications are broad, and future studies could integrate $\mathrm{Cu}-\mathrm{nZeo}$ into relevant final forms that are usable in practice.

Fe-nZeo and Fe-mZeo were also incubated with midlogarithmic phase MRSA and determined to be ineffective as an antimicrobial. Even at very high concentrations $(10 \mathrm{mg}$ $\mathrm{mL}^{-1}$ ), Fe-nZeo and Fe-mZeo slightly reduced MRSA viability, but failed to demonstrate bactericidal activity (Table 1; Fig. 7). These results were consistent with the iron ion release experiments, which showed that iron released from the Fe-nZeo or FemZeo was undetectable (Fig. 4).

Table 2 Antimicrobial effects of the ion-exchanged zeolites

\begin{tabular}{lllll}
\hline Sample & $\begin{array}{l}2 \mathrm{~h} \\
\text { MBC saline }\left(\mu \mathrm{g} \mathrm{mL} \mathrm{mL}^{-1}\right)\end{array}$ & $\begin{array}{l}\text { Ion equivalency } \\
(\mathrm{mM})\end{array}$ & $\begin{array}{l}24 \mathrm{~h} \\
\text { MBC CAMHB }\left(\mu \mathrm{g} \mathrm{mL}^{-1}\right)\end{array}$ & $\begin{array}{l}\text { Ion equivalency }^{a} \\
\left(\mathrm{mM}^{a}\right)\end{array}$ \\
\hline Zn-nZeo & 512 & 0.572 & $>2048$ & $>2.287$ \\
Zn-mZeo & 512 & 0.705 & $>2048$ & $>2.819$ \\
Cu-nZeo & 32 & 0.042 & $>2048$ & $>2.675$ \\
Cu-mZeo & 64 & 0.113 & $>2048$ & $>3.610$ \\
Fe-nZeo & $>10000$ & $>9.311$ & $>2048$ & $>1.907$ \\
Fe-mZeo & $>10000$ & $>13.072$ & $>2048$ & $>2.677$
\end{tabular}

${ }^{a} \mathrm{MBC}$ value based on the net metal ion content in mole.

(a)

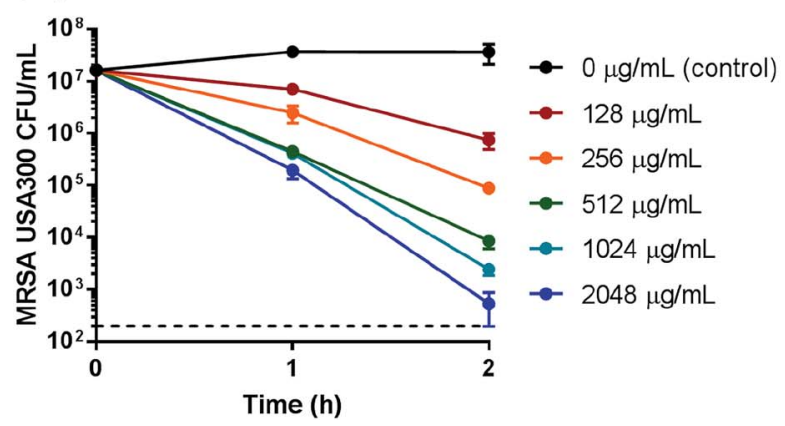

(b)

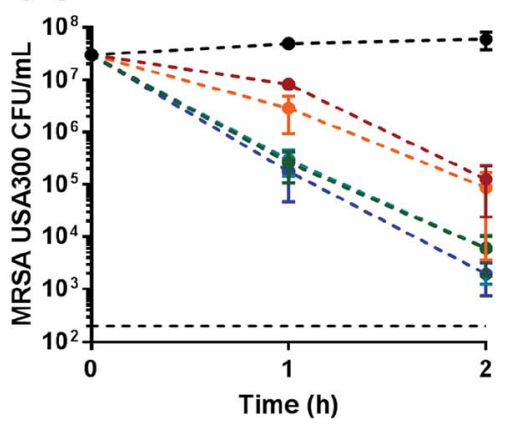

Fig. 5 Killing kinetics of MRSA USA300 after exposure to (a) Zn-nZeo or (b) Zn-mZeo. Zn-nZeo and Zn-mZeo were subjected to two-fold dilutions (2048-128 $\mu \mathrm{g} \mathrm{mL}^{-1}$ ), and suspensions were incubated in saline for $2 \mathrm{~h}$. The hatched line signifies the limit of detection. 
(a)

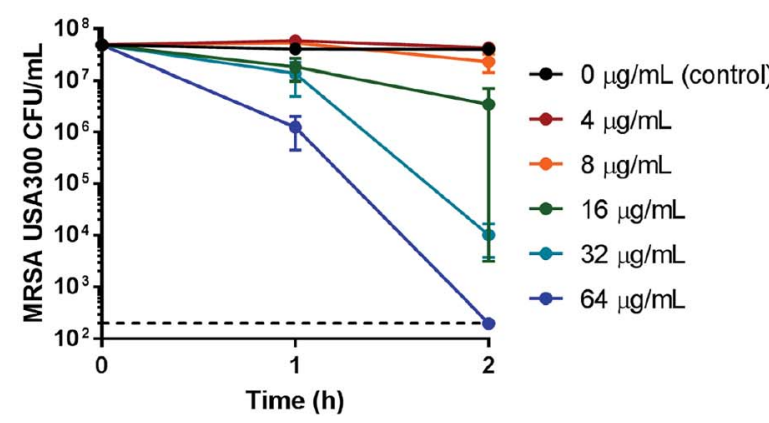

(b)

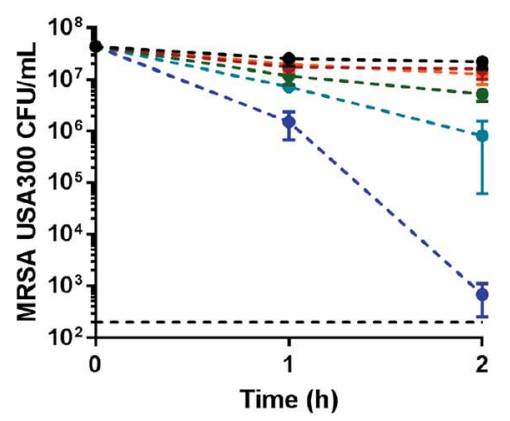

Fig. 6 Killing kinetics of MRSA USA300 after exposure to (a) Cu-nZeo or (b) Cu-mZeo. Cu-nZeo and Cu-mZeo were subjected to two-fold dilutions $\left(64-4 \mu \mathrm{g} \mathrm{mL}^{-1}\right)$, and suspensions were incubated in saline for $2 \mathrm{~h}$. The hatched line signifies the limit of detection.

(a)

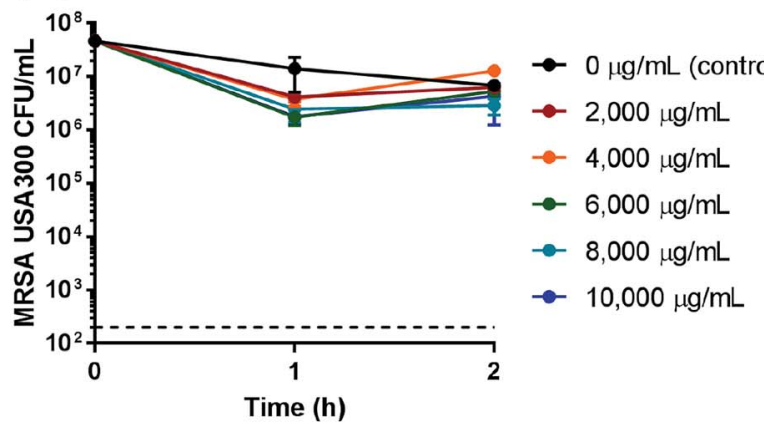

(b)

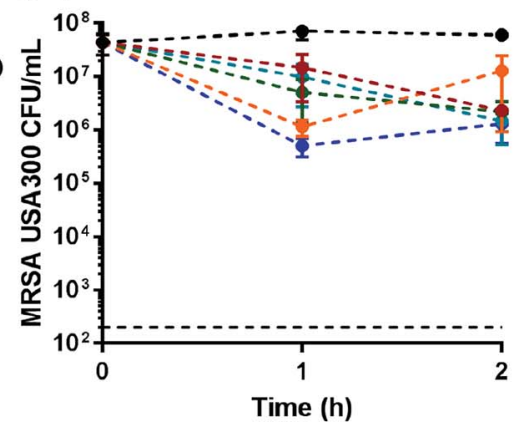

Fig. 7 MRSA USA300 viability after exposure to (a) Fe-nZeo or (b) Fe-mZeo. Fe-nZeo and Fe-mZeo were subjected to decreasing concentrations (10 000-2000 $\mathrm{g} \mathrm{mL}^{-1}$ ), and suspensions were incubated in saline for $2 \mathrm{~h}$. The hatched line signifies the limit of detection.

Overall, the ion release performance of the ion-exchanged zeolites is directly correlated with the antibacterial efficacy of the materials. $\mathrm{Cu}-\mathrm{nZ}$ Zeo releases the $\mathrm{Cu}$ ions twice as much as $\mathrm{Cu}-\mathrm{mZeo}$ and thus the MBC of the former is only a half of the value of the latter. That is, only one half amount of $\mathrm{Cu}$-nZeo is required to achieve the antibacterial efficacy of Cu-mZeo. Considering the positive effect of the nanoscopic morphology of the nZeo on the ion release performance, the superior antibacterial efficacy of Cu-nZeo must be due to the high surface area and small primary particle size of nZeo. For Zn, however, the effect is not as drastic because the ion release kinetics would not be greatly dissimilar between Zn-nZeo and Zn-mZeo due to the weak interactions between $\mathrm{Zn}$ ions and the zeolite framework (see above).

\section{Concluding remarks}

Comprehensive and comparative studies on porosity, morphology, ion release properties, and antibacterial performances are reported for zinc, copper, and iron ion-exchanged nanostructured zeolite $\mathrm{X}$ alongside microsized zeolite $\mathrm{X}$ ionexchanged with the same ions for comparison. Superior ion release properties were observed for both zinc and copper ionexchanged nanostructured zeolite $\mathrm{X}$, validating the importance of nanostructuring for enhanced ion diffusion through zeolite pore channels. The MBC for the copper ion-exchanged nanostructured zeolite $\mathrm{X}$ was half of the corresponding microsized zeolite $\mathrm{X}$, which indicates a superior performance of the nanostructured zeolite. However, antibacterial efficacy of zincand iron-exchanged nZeo did not show an improvement compared to the microsized zeolite counterparts. Our results established nanostructured zeolite $\mathrm{X}$ as a superior host material for metal ion-based antimicrobials with rapid metal ion release characteristics and rapid killing activity.

\section{Materials and methods}

\section{Synthesis of nanostructured zeolite $X$}

The nanostructured zeolite $\mathrm{X}$ was synthesized by first preparing a geopolymer resin with the composition of $3.0 \mathrm{Na}_{2} \mathrm{O}: 1.0 \mathrm{Al}_{2}$ $\mathrm{O}_{3}: 4.0 \mathrm{SiO}_{2}: 32.4 \mathrm{H}_{2} \mathrm{O}$. The geopolymer-resin was prepared by dissolving $4.555 \mathrm{~g}$ of $\mathrm{NaOH}$ pellets (Sigma Aldrich) and $11.711 \mathrm{~g}$ of water glass (Sigma Aldrich) in deionized (DI) water $(8.190 \mathrm{~g}$ ), prior to the addition of $5.735 \mathrm{~g}$ of metakaolin (MetaMax® from BASF). After stirring with a mechanical mixer (IKA ${ }^{\circledR}$ RW 60 digital mixer) at $800 \mathrm{rpm}$ for $40 \mathrm{~min}$, the visually homogeneous and free-flowing geopolymer resin was obtained. Into the resin, $15 \mathrm{~mL}$ of canola oil (J. M. Smucker Company, Crisco ${ }^{\circledR}$ ) was added and stirred for another $10 \mathrm{~min}$. The resin-oil mixture was then poured into $50 \mathrm{~mL}$ polypropylene tubes and tightly closed, 
and placed in a laboratory oven at $90{ }^{\circ} \mathrm{C}$ for $36 \mathrm{~h}$. After heating, the product, exhibiting a paste consistency, was removed from the tubes and washed with hot $\left(90^{\circ} \mathrm{C}\right)$ DI water through several cycles of centrifugation and redispersion in a large amount of the hot water until the $\mathrm{pH}$ of the filtrate reached about 8. The final product was collected, then dried in a laboratory oven at $90{ }^{\circ} \mathrm{C}$ overnight and stored in sealed glass vials at room temperature for further use.

\section{Preparation of zinc, copper, and iron ion-exchanged zeolites}

Zeolites, nZeo, or reference mZeo (13X, Sigma-Aldrich) (1 g) were suspended in $150 \mathrm{~mL}$ of UV-irradiated, nanopure, DI water in a $250 \mathrm{~mL}$ beaker. A $0.01 \mathrm{M}$ nitric acid solution was gradually added to the suspension until the $\mathrm{pH}$ reached $6.5,5.5$, or 7.0 for $\mathrm{Zn}^{2+}, \mathrm{Cu}^{2+}$, or $\mathrm{Fe}^{2+}$ ions, respectively, to avoid metal hydroxide precipitates. The metal ion solutions were prepared by dissolving $1 \mathrm{~g}$ of $\mathrm{Zn}\left(\mathrm{NO}_{3}\right)_{2} \cdot 6 \mathrm{H}_{2} \mathrm{O}\left(99 \%\right.$, Alfa Aesar), $\mathrm{Cu}(\mathrm{Ac})_{2} \cdot \mathrm{H}_{2} \mathrm{O}$ (>99.0\%, Sigma-Aldrich), or $\mathrm{FeSO}_{4} \cdot 7 \mathrm{H}_{2} \mathrm{O}$ (98\%, Alfa Aesar) into $50 \mathrm{~mL}$ DI water, followed by $\mathrm{pH}$ adjustment to 5.5 by gradual addition of $0.01 \mathrm{M}$ nitric acid. For the ion exchange reaction, the metal ion solutions were added to the zeolite suspensions and the mixtures were stirred gently for $24 \mathrm{~h}$. 10-20 mL of the suspension was taken out and was filtered with a syringe membrane filter with a polytetrafluoroethylene (PTFE) membrane $(0.45 \mu \mathrm{m})$, and the filtrate was collected for elemental analysis. The ion-exchanged zeolite particles were collected from the rest of the suspension by at least five cycles of repetitive centrifugation and resuspension in a copious amount of DI water, and dried overnight at $90{ }^{\circ} \mathrm{C}$. Particularly, the iron ion exchange was carried out with nitrogen-purged DI water in a nitrogen-filled glove bag to minimize the oxidation of ferrous $\left(\mathrm{Fe}^{2+}\right)$ ions to ferric $\left(\mathrm{Fe}^{3+}\right)$ ions which has a detrimental effect on ion exchange process. The product exhibited a slightly yellowish color, indicating a partial oxidation of the ferrous ions. The contents (loadings) of the transition metals in the ionexchanged zeolites were estimated from the difference between the amount of metal ions before and after ion exchange present in solution. The amounts of metal ions left in solution were calculated by multiplying the solution volume by the transition metal ion concentrations in the filtrates measured with the inductively coupled plasma-optical emission spectrometry (ICP-OES) (Thermo Scientific iCAP 6300 spectrometer).

\section{Characterization of zinc, copper, and iron ion-exchanged zeolites}

Powder X-ray diffraction (PXRD) patterns of the $\mathrm{Cu}$ - and $\mathrm{Zn}$ zeolite samples were collected on Bruker D8 powder X-ray diffractometer with $\mathrm{Cu} \mathrm{K} \alpha$ radiation with a wavelength of $1.5406 \AA$, and the PXRD patterns of Fe-zeolites were collected on Siemens $\mathrm{D} 5000$ powder diffractometer with Co $\mathrm{K} \alpha$ radiation with a wavelength of $1.7902 \AA$ at a scan speed of $2.0^{\circ} \mathrm{min}^{-1}$ and a step size of $0.04^{\circ}$. Scanning electron microscopy (SEM) images of powdered samples were collected using an XL30 environmental FEG (FEI) microscope operating at $15 \mathrm{kV}$ acceleration voltage. Brunauer-Emmett-Teller (BET) surface areas were estimated with a Micrometrics ASAP 2020 volumetric adsorption analyzer with nitrogen as the adsorbate at $77 \mathrm{~K}$. Prior to the analysis, samples (300 mg) were degassed at $300{ }^{\circ} \mathrm{C}$ for $10 \mathrm{~h}$ under vacuum until a residual pressure of $\leq 10 \mu \mathrm{m} \mathrm{Hg}$ was reached. Specific surface areas were determined from the BET equation. The $t$-plot method was used to distinguish the micropores from the mesopores in the samples and to calculate the external surface areas. The mesopore volumes were calculated by subtracting the micropore volume from the total pore volume. Mesopore size distributions were obtained using the Barrett-Joyner-Halenda $(\mathrm{BJH})$ method assuming a cylindrical pore model. ${ }^{39}$

\section{Zinc, copper, and iron ion release experiments}

In a typical experiment, $45 \mathrm{mg}$ of metal ion-exchanged zeolites was dispersed into $45 \mathrm{~mL}$ saline $(0.9 \% \mathrm{NaCl}$; w/v) to achieve a concentration of $1 \mathrm{mg} \mathrm{mL}^{-1}$. While the mixture was agitated at $600 \mathrm{rpm}$, a $10 \mathrm{~mL}$ aliquot was taken out after $3 \mathrm{~min}, 1 \mathrm{~h}$, and $2 \mathrm{~h}$. Each aliquot was filtered with a syringe membrane filter with a polytetrafluoroethylene (PTFE) membrane $(0.45 \mu \mathrm{m})$, and the filtrate was analyzed by ICP-OES. Dynamic light scattering experiments confirmed that zeolite particles were not present in the filtrates after the filtration process.

\section{Bacterial strains and growth conditions}

MRSA USA300 LAC (received from Dr Juliane BubeckWardenburg, University of Chicago, Chicago, IL), the most commonly isolated community-associated MRSA strain in the U.S., ${ }^{40}$ was grown in trypticase soy broth (TSB) or on trypticase soy agar (TSA). Cultures were grown from frozen glycerol stocks for $18 \mathrm{~h}$ with gentle mixing at $37^{\circ} \mathrm{C}$. Saturated cultures were then diluted $1: 40$ in TSB and grown to exponential phase by gentle mixing at $37^{\circ} \mathrm{C}$ for $2.5 \mathrm{~h}\left(\mathrm{OD}_{600}=0.3-0.4\right)$.

\section{Antibacterial susceptibility testing of ion-exchanged zeolites in saline}

In all the antibacterial susceptibility testing experiments, a negative control consisting of Na-nZeo or Na-mZeo was used to ensure that antibacterial activity was caused by release of metal ions instead of the zeolites themselves. All the samples were sterilized with $180{ }^{\circ} \mathrm{C}$ dry heat for $3 \mathrm{~h}$ prior to the testing. Exponential phase MRSA cultures were resuspended in sterile saline $(0.9 \% \mathrm{NaCl} ; \mathrm{w} / \mathrm{v})$. MRSA cells were then adjusted to a concentration of $3-5.5 \times 10^{7} \mathrm{CFU} \mathrm{mL}^{-1}\left(\mathrm{OD}_{600}=0.12\right)$ and mixed with varying amounts of the ion-exchanged zeolites. Suspensions of MRSA and ion-exchanged zeolites were incubated at $37{ }^{\circ} \mathrm{C}$ with gentle agitation. After $1 \mathrm{~h}$ and $2 \mathrm{~h}$, samples were serially diluted and plated on TSA to determine the minimum bactericidal concentration (MBC; $\geq 99.9 \%$ reduction in $\left.\mathrm{CFU} \mathrm{mL}^{-1}\right)$.

\section{Broth microdilution antibacterial susceptibility test of ion- exchanged zeolites}

A microtiter plate containing CAMHB with two-fold serial dilutions of ion-exchanged zeolites (2048 to $8 \mu \mathrm{g} \mathrm{mL}{ }^{-1}$ ) or vancomycin (positive control) was prepared. After addition of 
exponential phase MRSA $\left(1-3 \times 10^{5} \mathrm{CFU} \mathrm{mL}^{-1}\right)$, the microtiter plate was incubated at $37^{\circ} \mathrm{C}$ for $24 \mathrm{~h}$. The MBC was determined by plating samples onto Mueller Hinton agar and incubating plates at $37^{\circ} \mathrm{C}$ overnight.

\section{Conflicts of interest}

There are no conflicts to declare.

\section{Acknowledgements}

This research was supported by Public Health Service grant AI121733 awarded to S. E. H. and D.-K. S. from the NIH National Institute of Allergy and Infectious Diseases. We gratefully acknowledge the use of facilities within the LeRoy Eyring Center for Solid State Science at Arizona State University as well as BASF for their donation of metakaolin.

\section{References}

1 S. Mintova, M. Jaber and V. Valtchev, Chem. Soc. Rev., 2015, 44, 7207-7233.

2 A. A. Alswat, M. B. Ahmad, T. A. Saleh, M. Z. B. Hussein and N. A. Ibrahim, Mater. Sci. Eng., C, 2016, 68, 505-511.

3 A. A. Alswat, M. B. Ahmad, M. Z. Hussein, N. A. Ibrahim and T. A. Saleh, J. Mater. Sci. Technol., 2017, 33, 889-896.

4 A. A. Alswat, M. B. Ahmad and T. A. Saleh, Colloid Interface Sci. Commun., 2017, 16, 19-24.

5 A. P. Ingle, N. Duran and M. Rai, Appl. Microbiol. Biotechnol., 2014, 98, 1001-1009.

6 J. A. Lemire, J. J. Harrison and R. J. Turner, Nat. Rev. Microbiol., 2013, 11, 371-384.

7 C. C. Otto and S. E. Haydel, PLoS One, 2013, 8, e64068.

8 B. Simoncic and D. Klemencic, Text. Res. J., 2016, 86, 210223.

9 T. A. Saleh, M. M. Al-Shalalfeh and A. A. Al-Saadi, Sci. Rep., 2016, 6, 32185.

10 S. U. Khan, T. A. Saleh, A. Wahab, M. H. U. Khan, D. Khan, W. U. Khan, A. Rahim, S. Kamal, F. U. Khan and S. Fahad, Int. J. Nanomed., 2018, 13, 733.

11 D. W. Breck, Zeolite Molecular Sieves. Structure, Chemistry, and Use, Krieger, Malabar, Florida, 1974.

12 S. Demirci, Z. Ustaoglu, G. A. Yilmazer, F. Sahin and N. Bac, Appl. Biochem. Biotechnol., 2014, 172, 1652-1662.

13 L. Ferreira, J. F. Guedes, C. Almeida-Aguiar, A. M. Fonseca and I. C. Neves, Colloids Surf., B, 2016, 142, 141-147.

14 B. Galeano, E. Korff and W. L. Nicholson, Appl. Environ. Microbiol., 2003, 69, 4329-4331.

15 A. Top and S. Ulku, Appl. Clay Sci., 2004, 27, 13-19.

16 S. Chen, J. Popovich, N. Iannuzo, S. E. Haydel and D.-K. Seo, ACS Appl. Mater. Interfaces, 2017, 9, 39271-39282.
17 A. Sirelkhatim, S. Mahmud, A. Seeni, N. H. M. Kaus, L. C. Ann, S. K. M. Bakhori, H. Hasan and D. Mohamad, Nano-Micro Lett., 2015, 7, 219-242.

18 P. Bonaventura, G. Benedetti, F. Albarede and P. Miossec, Autoimmun. Rev., 2015, 14, 277-285.

19 A. B. Lansdown, U. Mirastschijski, N. Stubbs, E. Scanlon and M. S. Agren, Wound Repair Regen., 2007, 15, 2-16.

20 M. Vincent, P. Hartemann and M. Engels-Deutsch, Int. J. Hyg. Environ. Health, 2016, 219, 585-591.

21 G. Grass, C. Rensing and M. Solioz, Appl. Environ. Microbiol., 2011, 77, 1541-1547.

22 C. Otto and S. Haydel, Microbial Pathogens and Strategies for Combating them: Science, Technology and Education, Badajoz, Spain, 2013, pp. 1169-1180.

23 L. Macomber and J. A. Imlay, Proc. Natl. Acad. Sci. U. S. A., 2009, 106, 8344-8349.

24 F. F. Xu and J. A. Imlay, Appl. Environ. Microbiol., 2012, 78, 07368-07311.

25 J. Redfern, K. Goldyn, J. Verran, R. Retoux, L. Tosheva and S. Mintova, Microporous Mesoporous Mater., 2017, 253, 233238.

26 D. H. Olson, J. Phys. Chem., 1970, 74, 2758-2764.

27 B. E. Warren, X-ray Diffraction, Courier Corporation, 1969.

28 D. Bae, S. Zhen and K. Seff, J. Phys. Chem. B, 1999, 103, 56315636.

29 R. t. Shannon, Acta Crystallogr., Sect. A: Cryst. Phys., Diffr., Theor. Gen. Crystallogr., 1976, 32, 751-767.

30 S. J. Gentry, N. W. Hurst and A. Jones, J. Chem. Soc., Faraday Trans. 1, 1979, 75, 1688-1699.

31 A. Zecchina, M. Rivallan, G. Berlier, C. Lamberti and G. Ricchiardi, Phys. Chem. Chem. Phys., 2007, 9, 3483-3499.

32 A. Maes and A. Cremers, J. Chem. Soc., Faraday Trans. 1, 1975, 71, 265-277.

33 A. Dyer and R. P. Townsend, J. Inorg. Nucl. Chem., 1973, 35, 3001-3008.

34 A. Dyer and S. A. I. Barri, J. Inorg. Nucl. Chem., 1977, 39, 10611063.

35 D. Pinkert and C. Limberg, Chem.-Eur. J., 2014, 20, 91669175.

36 B. McNicol and G. Pott, J. Catal., 1972, 25, 223-229.

37 R. Tekin and N. Bac, Microporous Mesoporous Mater., 2016, 234, 55-60.

38 L. Tosheva, A. Brockbank, B. Mihailova, J. Sutula, J. Ludwig, H. Potgieter and J. Verran, J. Mater. Chem., 2012, 22, 1689716905.

39 E. P. Barrett, L. G. Joyner and P. P. Halenda, J. Am. Chem. Soc., 1951, 73, 373-380.

40 F. C. Tenover and R. V. Goering, J. Antimicrob. Chemother., 2009, 64, 441-446. 